\title{
Recognition of English Characters Using Spiking Neural Networks
}

\author{
Amjad J. Humaidi ${ }^{\# 1}$, Thaer M. Kadhim ${ }^{* 2}$ \\ Control and System Engineering, University of Technology, Iraq, Baghdad \\ 1601116@uotechnology.edu.iq \\ Control and System Engineering, University of Technology, Iraq, Baghdad \\ 2cse.61012@uotechnology.edu
}

\begin{abstract}
Text Recognition is one of the active and challenging areas of research in the pattern recognition field. It covers many applications like automatic number plate recognition, bank cheques, aid of reading for blind, and hand-written document conversion into form of structural text. In the present work, the spiking neural network (SNN) model is utilized for the identification of characters in a character set. The structure of considered neural network consists of two layers with Izhekevich neurons. Remote Supervised Method (ReSuMe) has been used as a learning rule for training. Also, the recognition of English characters has been considered based on spike Neural Networks. The network could successfully recognize a set of $\mathbf{4 8}$ characters.
\end{abstract}

Keyword- Spiking Neural Network (SNN), Remote Supervised Method (ReSuMe), Artificial Neural Networks(ANN)

\section{INTRODUCTION}

Spiking neuron models lead to the rise of third generation of artificial neural networks(ANN) [1]. Such models add more realistic sense in neural simulation and invocation of the time concept. It has been shown that a considerable enhancement in image recognition speed can be reached by using pulse coding (versus rate coding) in biological neurons of the cortex [2]. Generally, a longer processing time is required by rate coding as the neuron could be fired after waiting for several spikes to come. Also, in case of pulse coding, the information based on timing order of incoming spikes can be encoded at faster rate.

The researchers in [3] and [4] have used spiking neural networks based on pulse coding for image recognition application. The model has been developed by Gupta could recognize a set of 48 characters with $3 \times 5$ pixel size. The model of integrate and fire spiking neuron network has been utilized for recognition and the spike timing based plasticity (STDP) has been used for training [5]. Thorpe used the same neuron model to examine the recognition, but with enlarged size of images [6]. Buonomano et al. have proposed a spiking neural model which can code the intensity of the input using relative firing time for the application of position invariant character recognition [7]. Presently, researchers in the field of character recognition systems showed a strong interest to find models which mimicking the biological nature neural networks. In other words, it would be useful to inspect for such systems (image recognition) the use of neuron models which are more identical to biological nature than integrate-and-fire model. Izhikevich stated that the integrate and fire model is far from mimicking the true biological nature of spiking neuron models. He notified that integrate-and-fire model cannot satisfy most essential features of cortical spiking neurons and thereby this model has to be avoided [8]. Izhikevich has set up a comparison analysis between 11 spiking neuron models in the aspects of biological accuracy and computational load [8]. He sorted the models according to their biological accuracy and showed that the first five most biologically accurate models are Hodgkin-Huxley, Izhikevich, Wilson, Hindmarsh-Rose, and Morris-Lecar models. It has been shown that the model of Hodgkin-Huxley is the most computationally intensive, while that of Izhikevich is the most computationally efficient.

In the present work, the spiking neuron network for character recognition is presented. The structure of neural network is a two-layer spiking neuron network based on biological-like Izhikevich neuron model. Also, the Remote Supervised Method (ReSuMe) is suggested for model training and the objective of neuron network is to recognize a set of 48 characters with a $5 \times 3$ pixel size.

\section{NEURON MODEL}

In 2003, a new spiking neuron model has been proposed by Izhikevich The model is characterized by second order differential equations [9]. It has been shown that the computations required by Izhikevich model is significantly fewer (13 flops per neuron update) than that needed by Hodgkin-Huxley model (1200 flops per neuron update) [8]. In spite of that Izhikevich model could successfully produce the responses of most types of neurons that have been used in biological experiments. As had been done by Izhikevich, the time step of $1 \mathrm{~ms}$ is utilized [9].

$$
\mathrm{dV} / \mathrm{dt}=0.04 \mathrm{~V}^{2}+5 \mathrm{~V}+140-\mathrm{u}+\mathrm{I}
$$


Subjected to the condition;

$$
\mathrm{du} / \mathrm{dt}=\mathrm{a}(\mathrm{bV}-\mathrm{u})
$$

$$
\text { and }\{\mathrm{u} \leftarrow \mathrm{u}+\mathrm{d}\} \quad \text { if } \mathrm{V} \geq 30 \text {, then }\{\mathrm{V} \leftarrow \mathrm{c}\}
$$

The variables $\mathrm{V}$ and $\mathrm{u}$ denote the potential of neuron membrane and its recovery variable, respectively. The variable I refers to the total synaptic current. The portion $0.04 \mathrm{~V}^{2}+5 \mathrm{~V}+140$ has been acquired by fitting the spike initiation dynamics of cortical neurons such that the unit of variable $\mathrm{V}$ correspond to $\mathrm{mV}$ and unit of time correspond to ms. By suitable adjustment of fixed parameters (a, b, c, and d), Izhikevich model can produce the responses of most types of neurons shown in biological tests. The values of constants are listed in Appendix. The spikes obtained by this model are depicted in Fig. 1.



Fig. 1. Spikes generated by Izhikevich model

\section{III.CHARACTER RECOGNITION ALGORITHM}

In this algorithm, a two-layer spiking neural network structure based on Izhikevich model has been used for character recognition. The first layer of the network works as input neurons, while the second layer represents the output neurons. The suggested network has been trained to recognize the 48 different input images corresponding to 48 different characters as indicated in Fig. 2 . These 48 images correspond to upper case (A-Z) letters, eight Greek letters, 10 numerals (0-9) and four symbols. The characters corresponding to input images are given to the first layer of neurons (designated as level 1), such that each pixel of the image is presented to its corresponding individual input neuron. Therefore, the number of pixels in the input image is equivalent to the

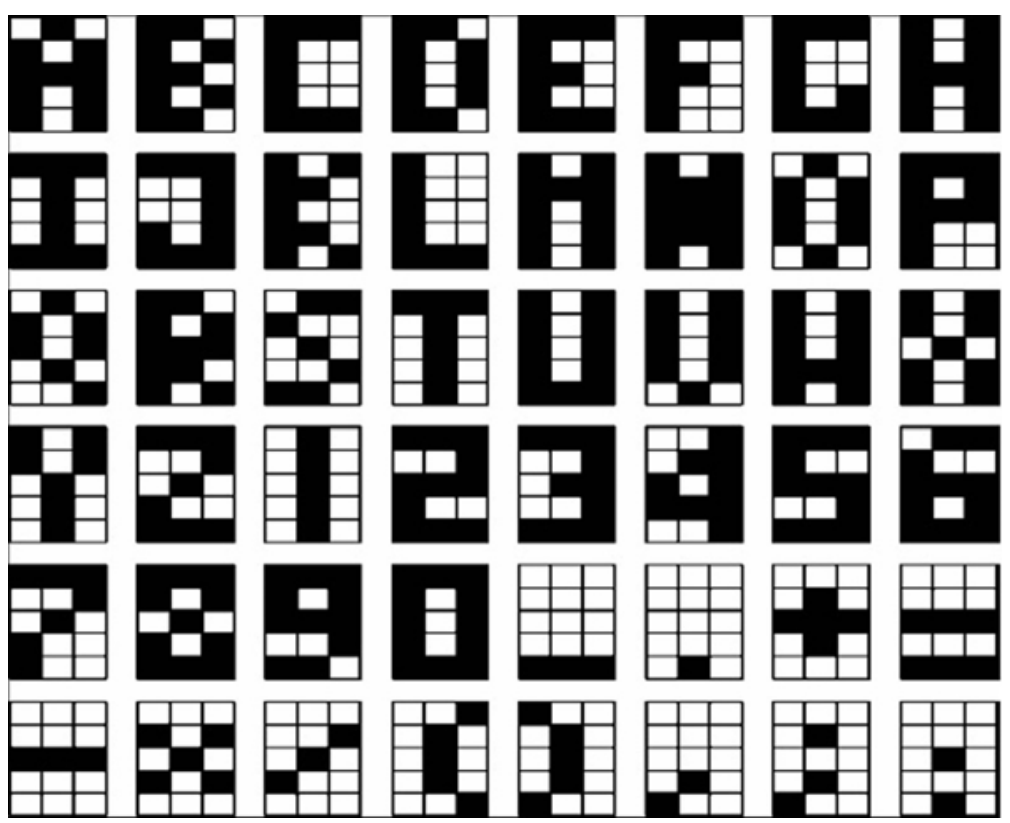

Fig. 2. Initial character test 
number of neurons in level 1. Moreover, the number of training images is equal to number of neurons at second layer, which is referred to as level 2. This is because each neuron of second layer (level 2) has been encoded to fire only when it recognizes its corresponding image. Each neuron of level 1 is linked to every neuron of level 2. A prototype of this neural network structure is depicted in Fig. 3.

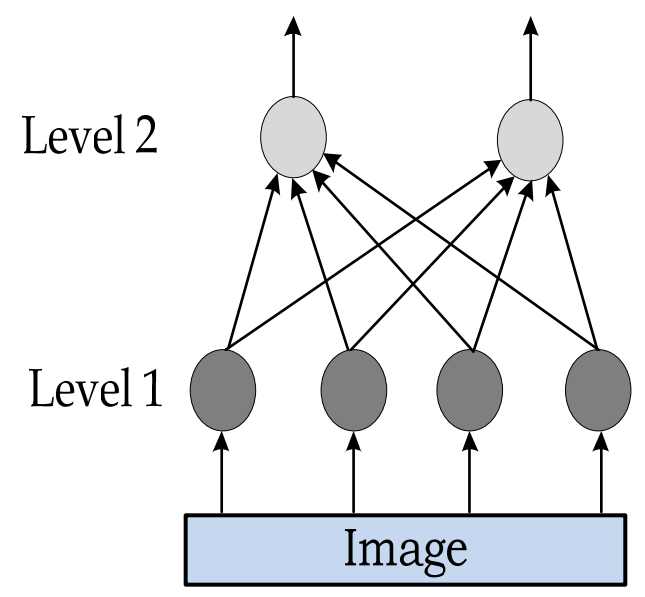

Fig. 3. The Neural Structure and Connection between the Neurons of Two Levels.

Membrane potential $V$ is evaluated by input current $I$ of each neuron. If this potential of membrane reaches a certain threshold level through the cycle, the neuron is promptly fired. Concerning the neuron of level 1 , the input current I of certain neuron is set to zero if its corresponding pixel in the input image is "off ". However, a constant current is fed to the input neuron if its corresponding input pixel of image is "on".

On the other hand, the input current to a certain neuron at level 2 is the equal to the sum of all individual currents coming from the connected neurons at level 1 . The expression of input current I (j) for neuron $\mathrm{j}$ at level 2 is described by Eq. (4),

$\mathrm{I}(j)=\sum_{\mathrm{i}} w(i, j) f(i)$

where $w$ is a weight matrix whose entries $w(i, j)$ is the input weight from neuron $i$ (at level 1 ) to neuron $j$ (at level 2). The function $f(i)$ shown in Eq. (4) is described as a firing vector whose value is equal to zero if the $i^{\text {th }}$ neuron of level 1 does not fire, and it is equal to one if the $i^{\text {th }}$ level 1 neuron does fire. Training process is responsible for determining the entries of the weight matrix $\mathrm{w}$ immediately after the process of driving the set of training images sequentially to the input neurons. The training process would produce a weight matrix, which cab utilized to evaluate the input current of each output neurons.

During the recognition phase, an input image is delivered to neurons of the level 1 and there is one output neuron will be fired after passing a certain number of cycles; indicating that the input image is identified. Based on the input image, the neurons at level 1 are evaluated during each cycle such that the firing vector is updated to specify which neurons are fired at that cycle. At the same cycle, the firing vector resulting from the last cycle is utilized to determine the value of input current I coming into each neuron at level 2. Based on input current values of neurons at level 2, their membrane potentials $V$ can be determined.

\section{IV.LEARNING METHOD}

The synaptic weights of the network in supervised training algorithms are updated iteratively such that the desired input/output mapping to the SNN is obtained [11]. The present work adopts the Remote Supervised Method (ReSuMe) rule for updating the weight of a synapse i. In Artificial Neural Networks, the basic principle of supervised learning method is based on synaptic weight adaptation $\Delta \mathrm{w}$, which is expressed in terms error function $\mathrm{e}=\left(\mathrm{y}_{\mathrm{d}}-\mathrm{y}_{\text {out }}\right)$ between the desired $\left(\mathrm{y}_{\mathrm{d}}\right)$ and the actual neuron output $\left(\mathrm{y}_{\text {out }}\right)[12]$ :

$\Delta \mathrm{w}_{\mathrm{i}}=\gamma \mathrm{x}_{\mathrm{i}}\left(\mathrm{y}_{\mathrm{d}}-\mathrm{y}_{\text {out }}\right)$

where $\gamma$ is a positive real-valued learning rate, $x_{i}$ is the input passing through synapse $i, y_{d}$ the desired neural output and $\mathrm{y}_{\text {out }}$ refer to the actual neural output. The weight updating rule indicated by Eq. (5) was commonly used for traditional neural networks.

In spike neural network, a series of spikes are transferred among neurons and the form of information exchange represented by Eq. (3) has to be explained. 
The supervised learning process consists of three types of participating neurons: input, learning and teacher neurons [12]. The set of the input neurons designated as $\mathrm{N}^{\mathrm{in}}=\left(\mathrm{N}_{1}^{\text {in }}, \mathrm{N}_{2}^{\text {in }}, \ldots \ldots ..\right)$ are the neurons responsible for activating the learning synapses. The set defined by $\mathrm{N}^{\mathrm{o}}=\left(\mathrm{N}_{1}^{\mathrm{o}}, \mathrm{N}_{2}^{\mathrm{o}}, \ldots\right)$ are the learning neurons and they invoke all neurons that receive signals from the learning synapses, which try to yield the target signals $S^{d}(t)$. Then, the signals $S^{d}(t)$ are submitted to the network by means of a set of the teacher neurons $N^{d}=\left(N_{1}^{d}, N_{2}^{d}, \ldots ..\right)$. Fig. 4 shows three types of neurons.

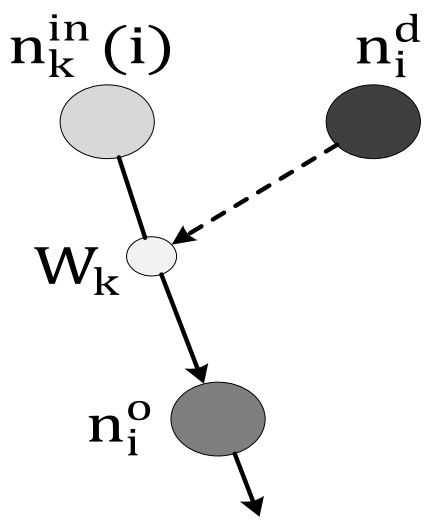

Fig. 4. Three neurons types

In Izhikevick models, the neurons are stimulated by increasing input current to the input neuron, therefore the currents of teacher neuron and learning neuron consider $y_{d}$ and $y_{\text {out }}$ respectively. Then, Eq. (5) can be written as:

$$
\Delta \mathrm{W}=\gamma \mathrm{I}_{\mathrm{i}}\left(\mathrm{I}_{\mathrm{j}}^{\mathrm{d}}-\mathrm{I}_{\mathrm{j}}^{\mathrm{o}}\right)
$$

The change in the synaptic strength between neurons $\mathrm{N}_{\mathrm{i}}^{\mathrm{in}}$ and $\mathrm{N}_{\mathrm{j}}^{\mathrm{o}}$ requires information about the synapse between neurons $\mathrm{N}_{\mathrm{i}}^{\text {in }}$ and $\mathrm{N}_{\mathrm{k}}^{\mathrm{d}}$

$\Delta \mathrm{w}_{\mathrm{ij}}=-\Delta \mathrm{w}_{\mathrm{ij}}+\Delta \mathrm{w}_{\mathrm{ik}}$ for excitory synapses

$$
\Delta \mathrm{w}_{\mathrm{ij}}=\Delta \mathrm{w}_{\mathrm{ij}}-\Delta \mathrm{w}_{\mathrm{ik}} \quad \text { for inhibitory synapses }
$$

\section{RESULT}

This work utilized the same set of characters used in the work referred by [13], which used a structure of traditional neural network and based on back propagation on massively parallel computer. The suggested Izhikevick model has been simulated and tested using MATLAB.

The spike neural network has been initialized and trained for the character set consists of only 12 characters ('A', 'B', 'C', 'D', 'F', 'L', 'U', 'T', '+', '-', '/, and ' $\times$ ') as shown in Fig. 5. For this case, the elements of network structure consist of 15 input neurons and 12 output neurons. In Tab.1, described in the appendix, lists the running parameters used in this work. The initial values of weights are set to random values, range between 0 and 1 , such that all output neurons would spike at the first iteration of training. During the training process, each character is presented to the spike neural structure sequentially one at a time. Through the application of Remote Supervised Method (ReSuMe) learning method, the elements of weight matrix would continuously change until the outputs of both the teacher neuron and learning neuron are identically equal and the network is said to be fully trained at this stage. 

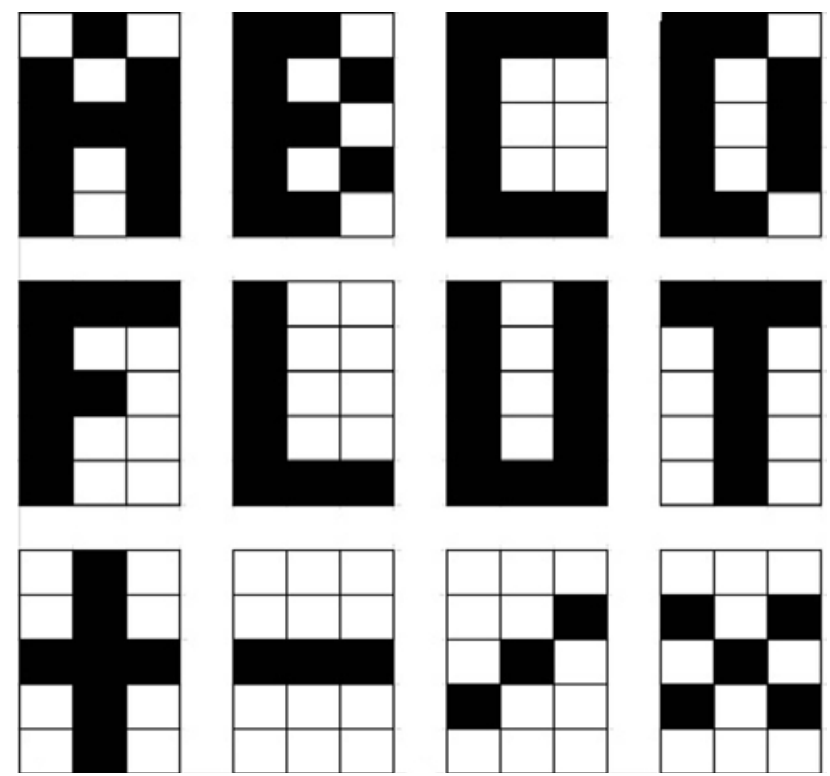

Fig.5 Initial character test

The responses of twelve trained neurons when fed with characters 'A', 'B', 'C', 'D', 'F', 'L', 'U', 'T', '+', '', ' $/$, and ' $\times$ ' are illustrated in Fig. 6 to Fig. 17. It is clear from the figures that there is a particular character responding to only one neuron, which is continuously spiking with spike-time interval of $100 \mathrm{~ms}$. Other Neurons show no spikes; as their membrane potentials are below the threshold value designated by $30 \mathrm{mV}$. Particularly, neurons $1,2,3,4,5,6,7,8,9,10,11$, and 12 respond to characters 'A', 'B', 'C', 'D', 'F', 'L', 'U', 'T', '+', '', ',', and ' $\times$ ' respectively
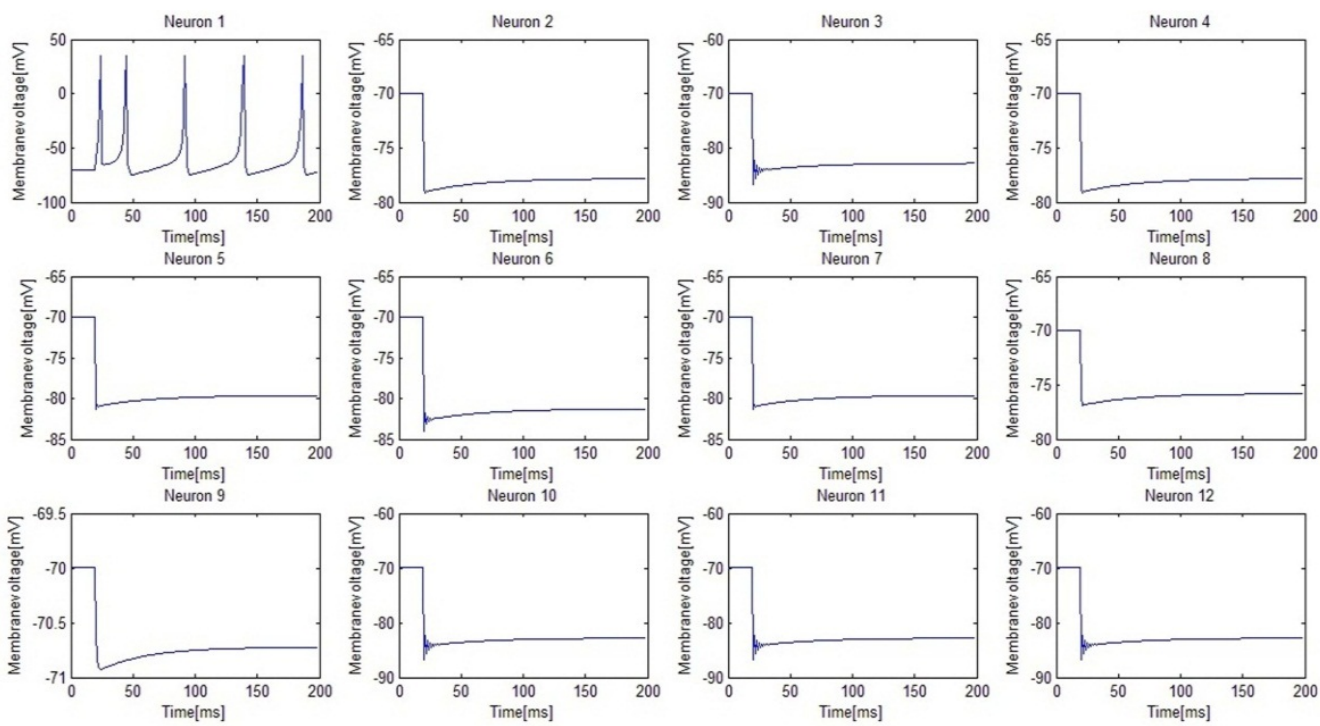

Fig.6 Behaviour of membrane voltages for character 'A' 

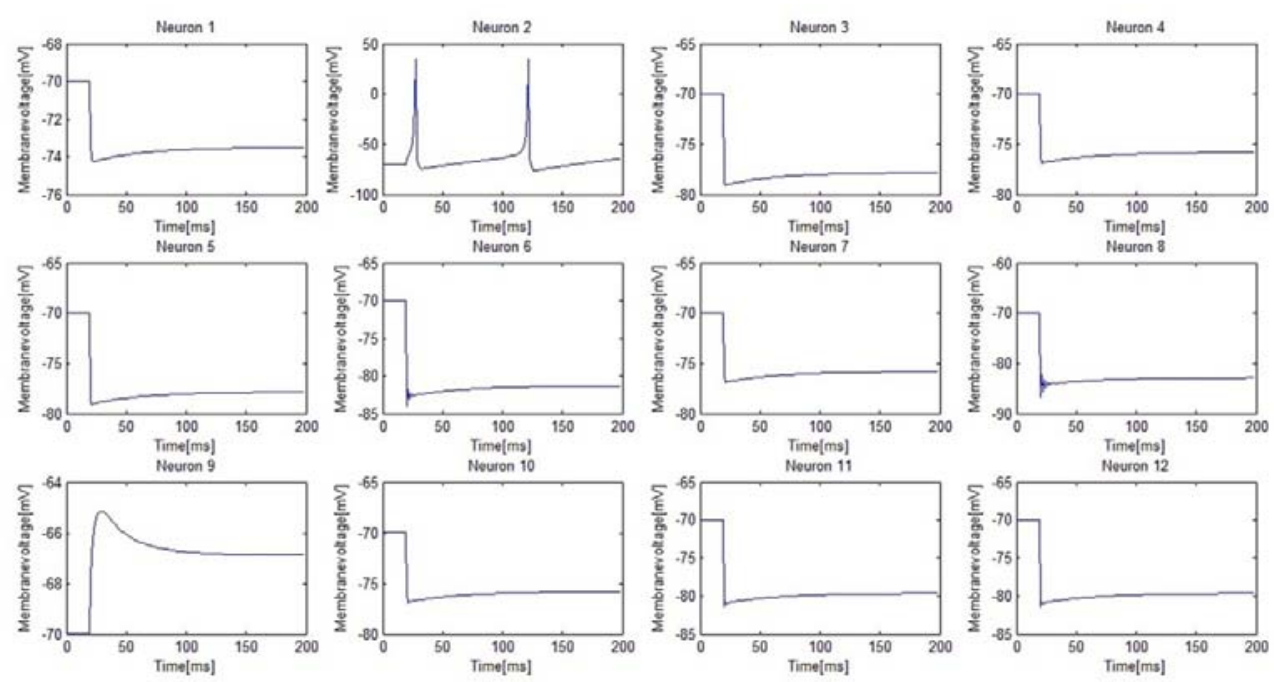

Fig.7 Behaviour of membrane voltages for character 'B'
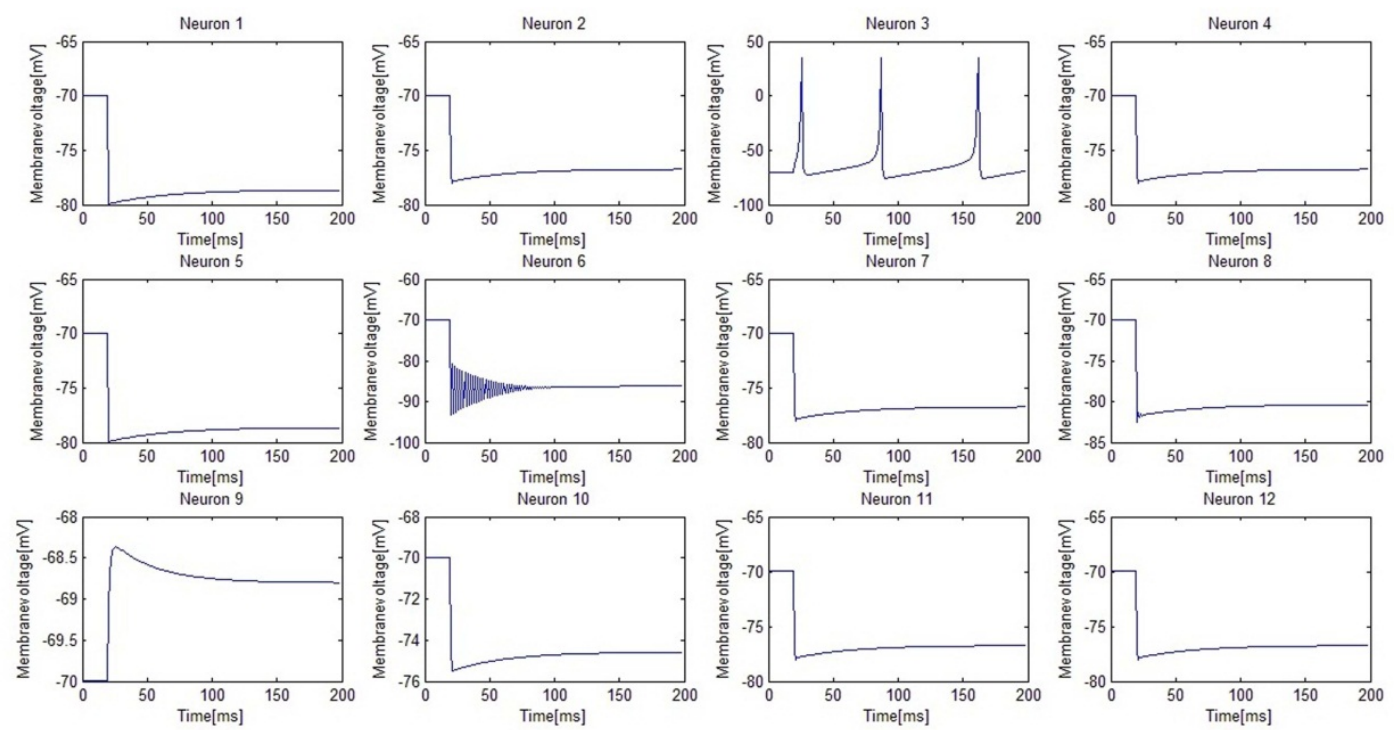

Fig.8 Behaviour of membrane voltages for character ' $\mathrm{C}$ '
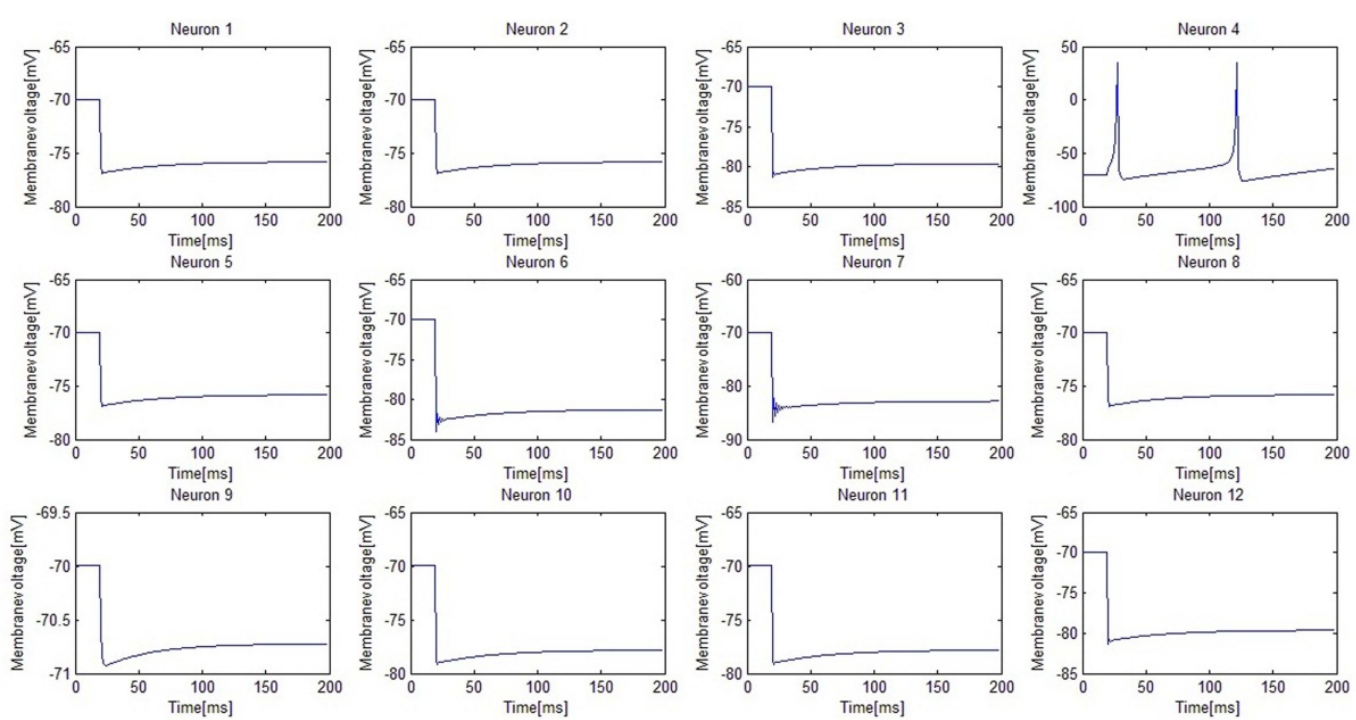

Fig.9 Behaviour of membrane voltages for character 'D' 

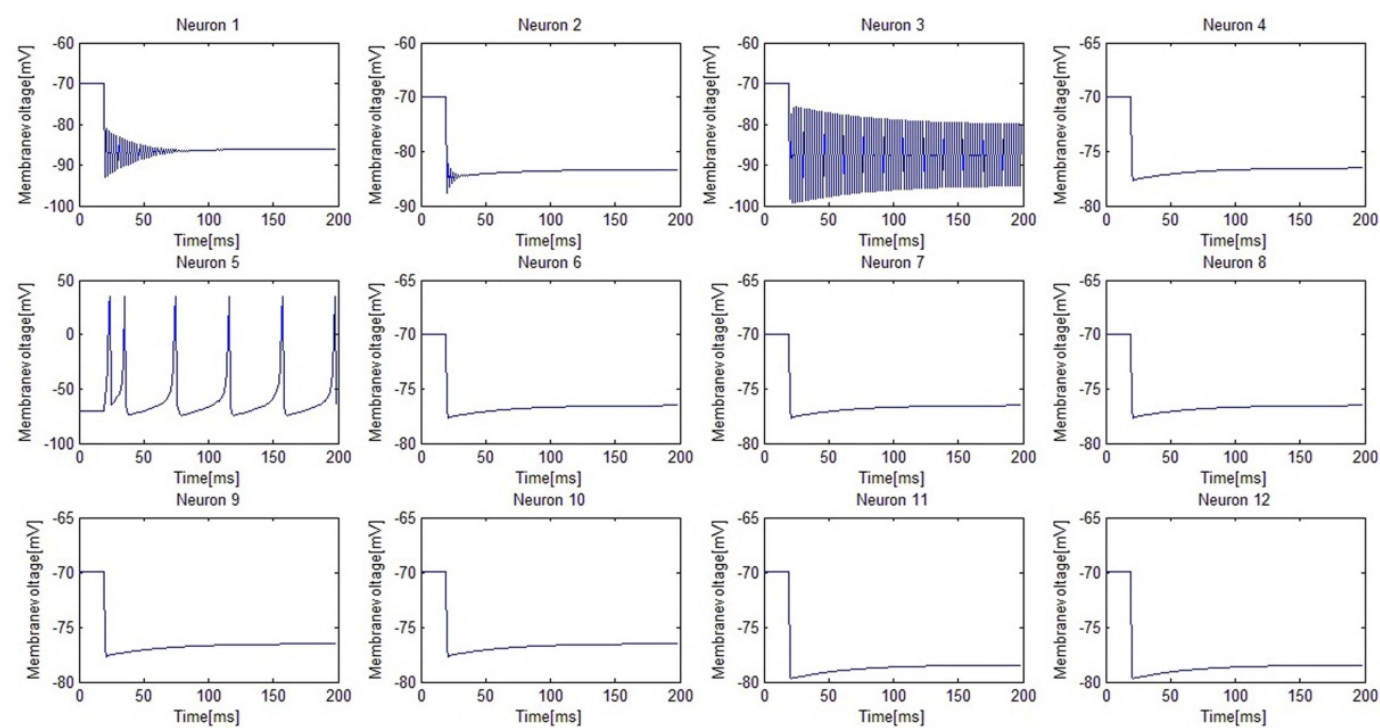

Fig.10 Behaviour of membrane voltages for character ' $\mathrm{F}$ '
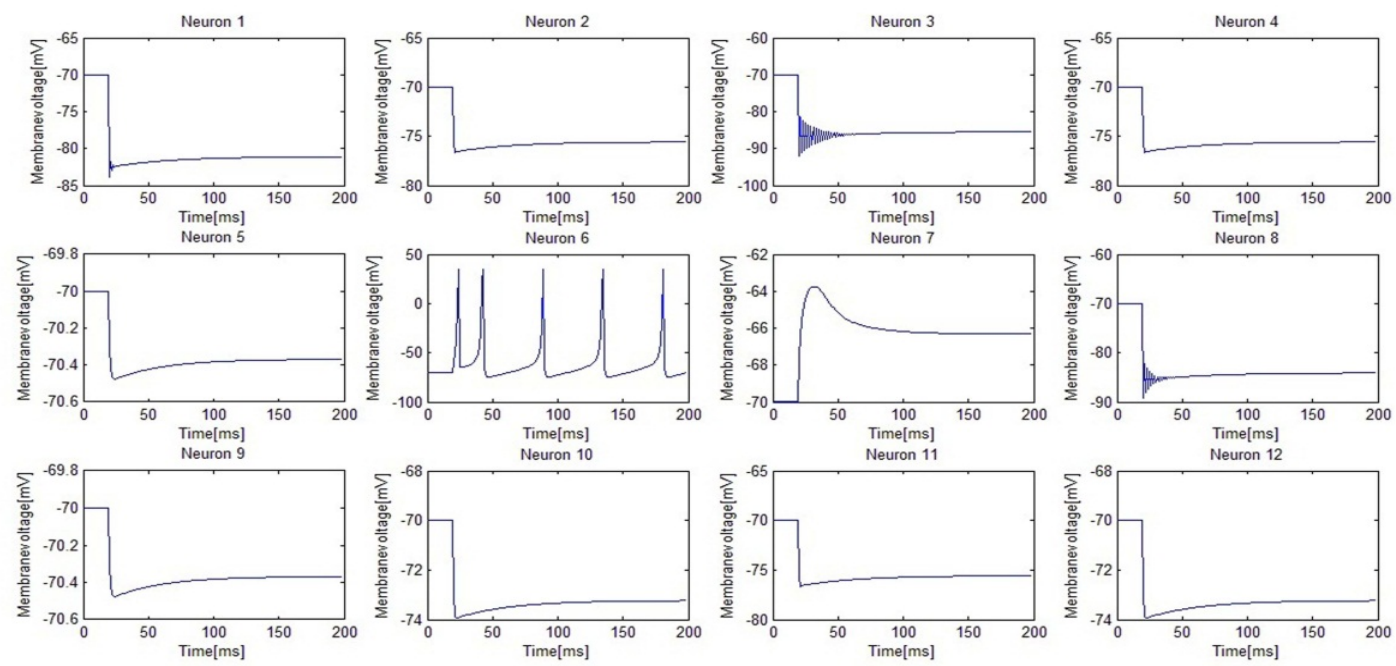

Fig.11 Behaviour of membrane voltages for character ' $\mathrm{L}$ '
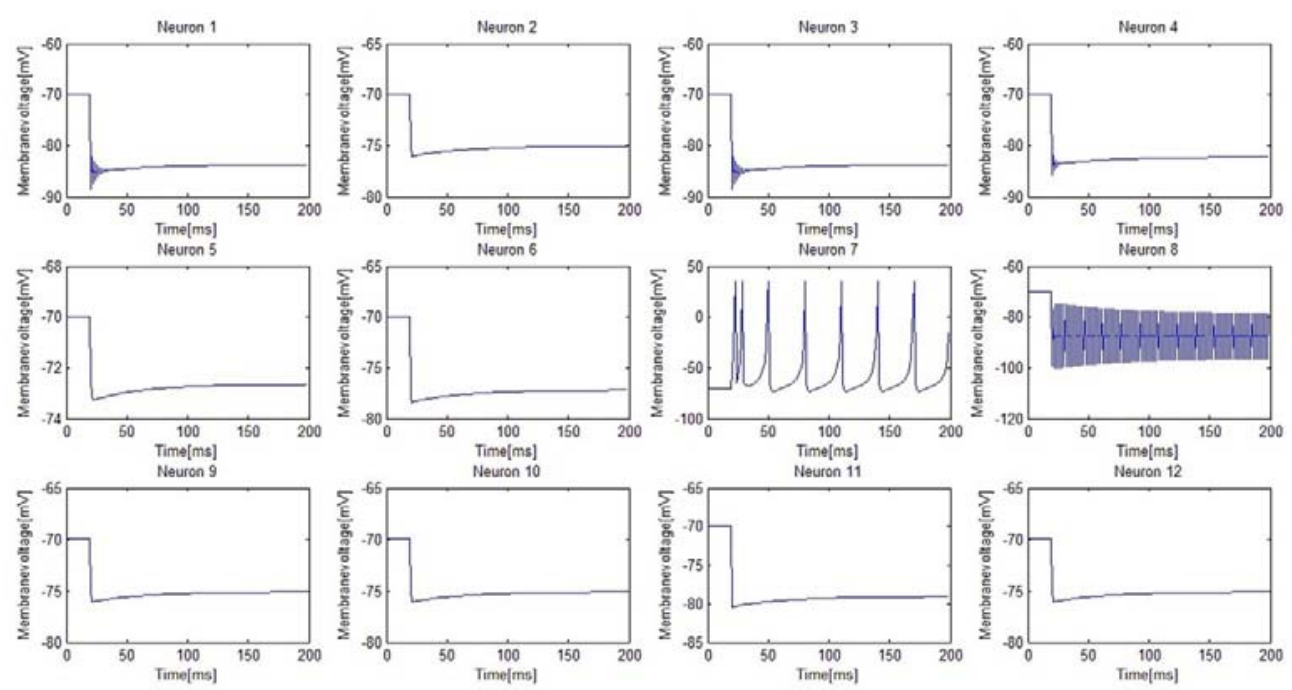

Fig.12 Behaviour of membrane voltages for character ' $U$ ' 

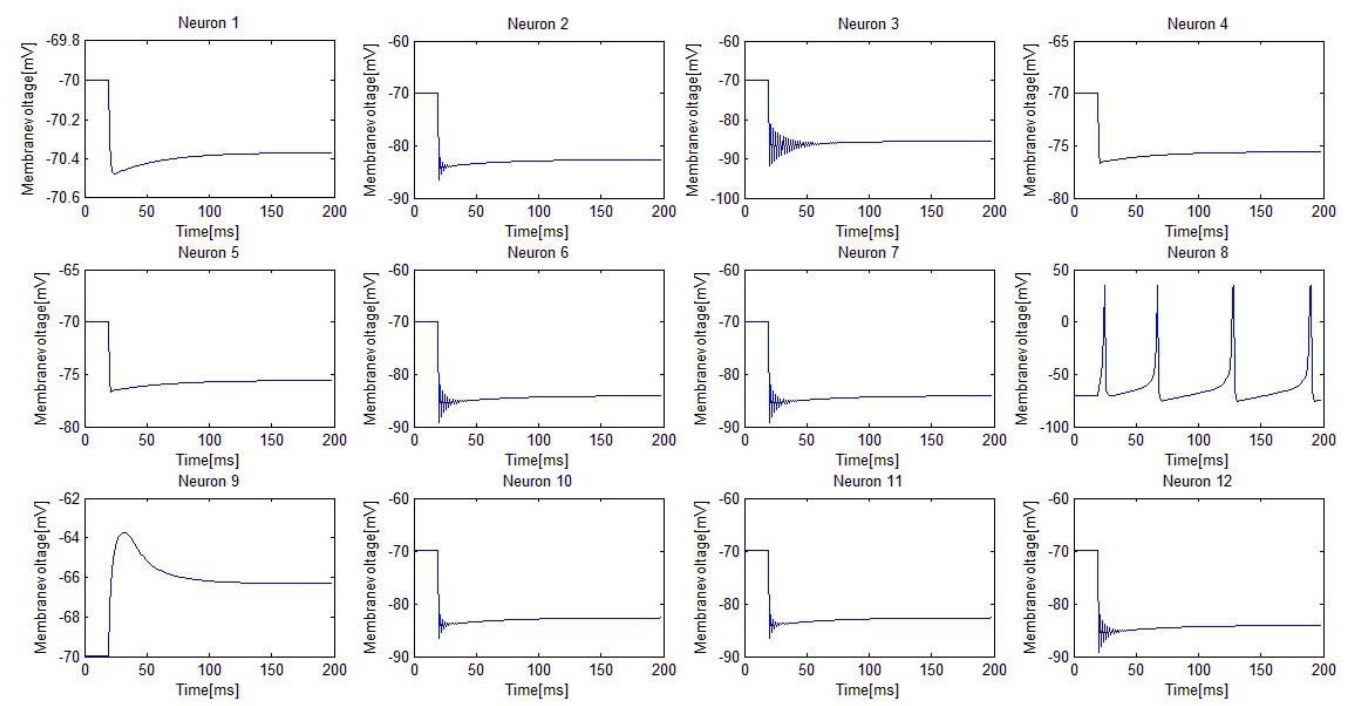

Fig.13 Behaviour of membrane voltages for character ' $\mathrm{T}$ '
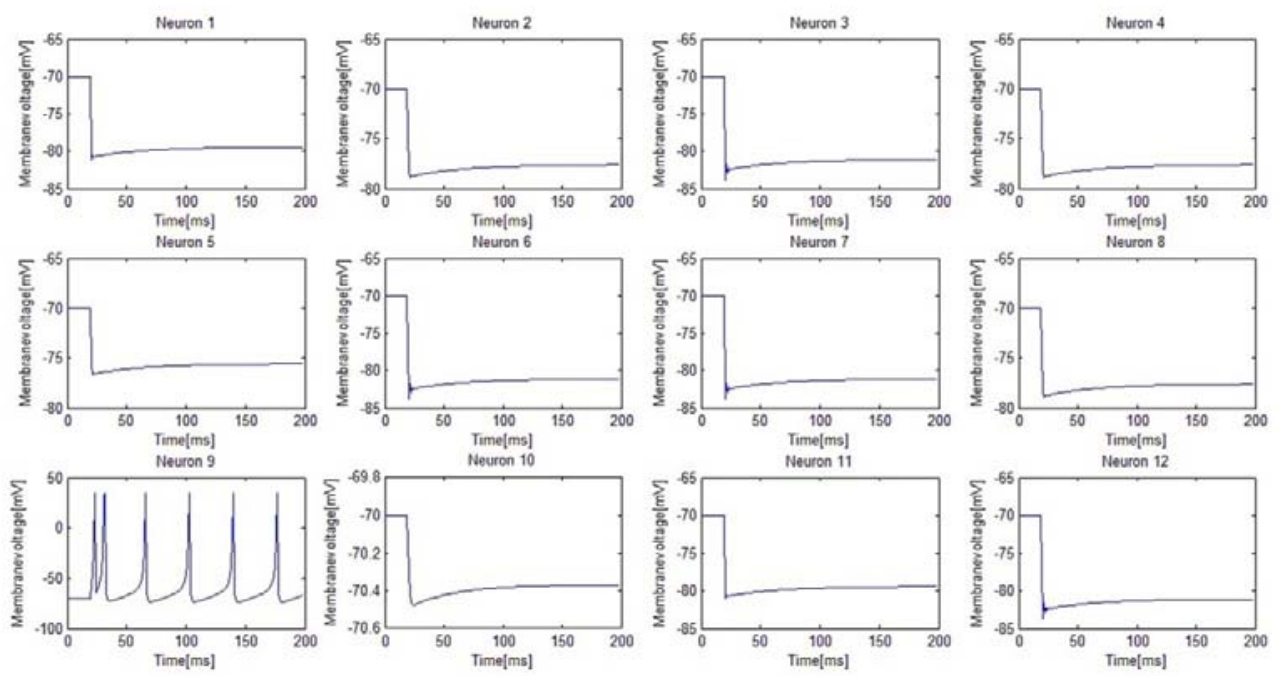

Fig.14 Behaviour of membrane voltages for character ' + '
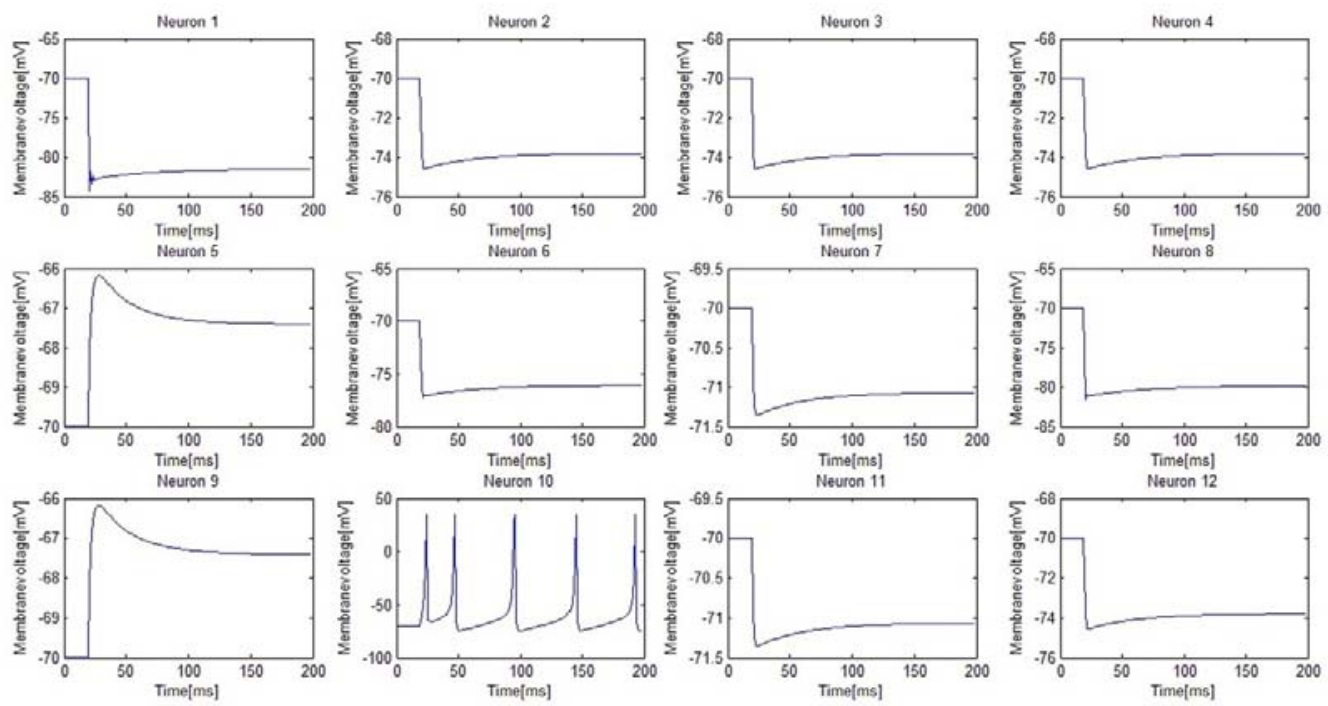

Fig.15 Behaviour of membrane voltages for character '-' 

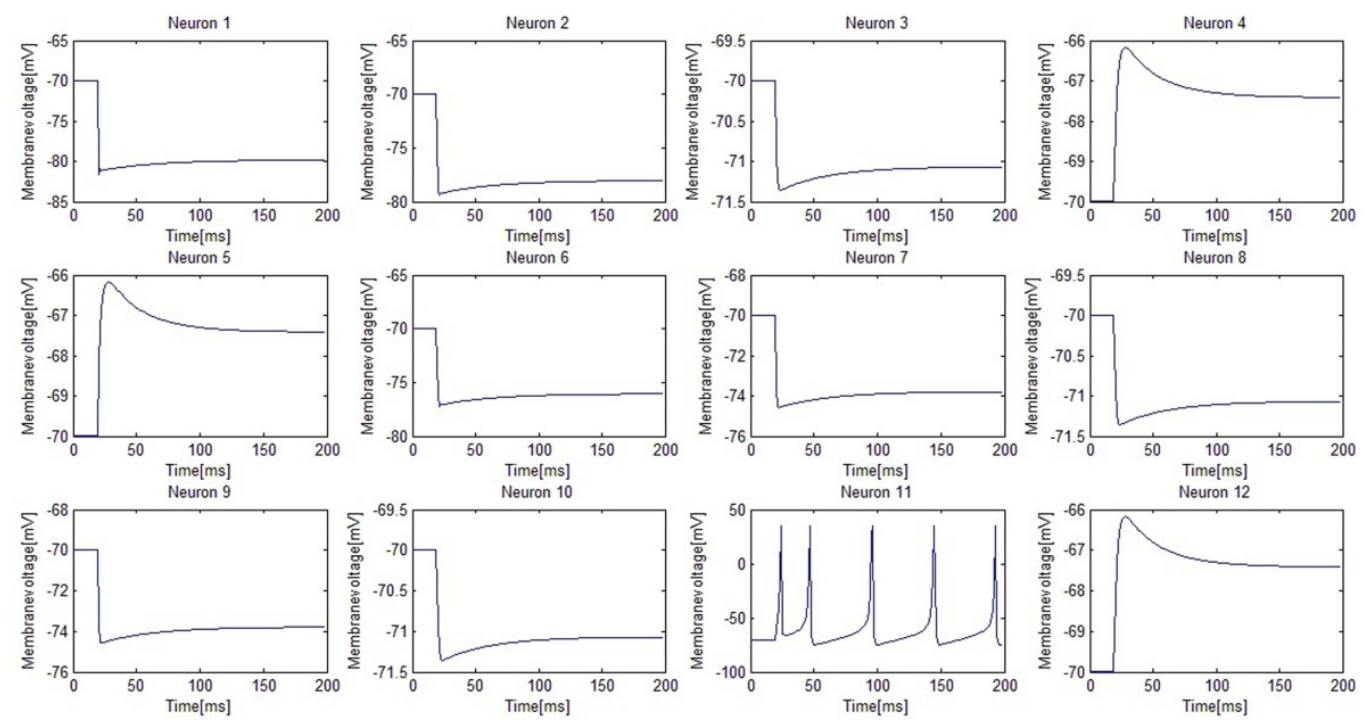

Fig.16 Behaviour of membrane voltages for character '/'
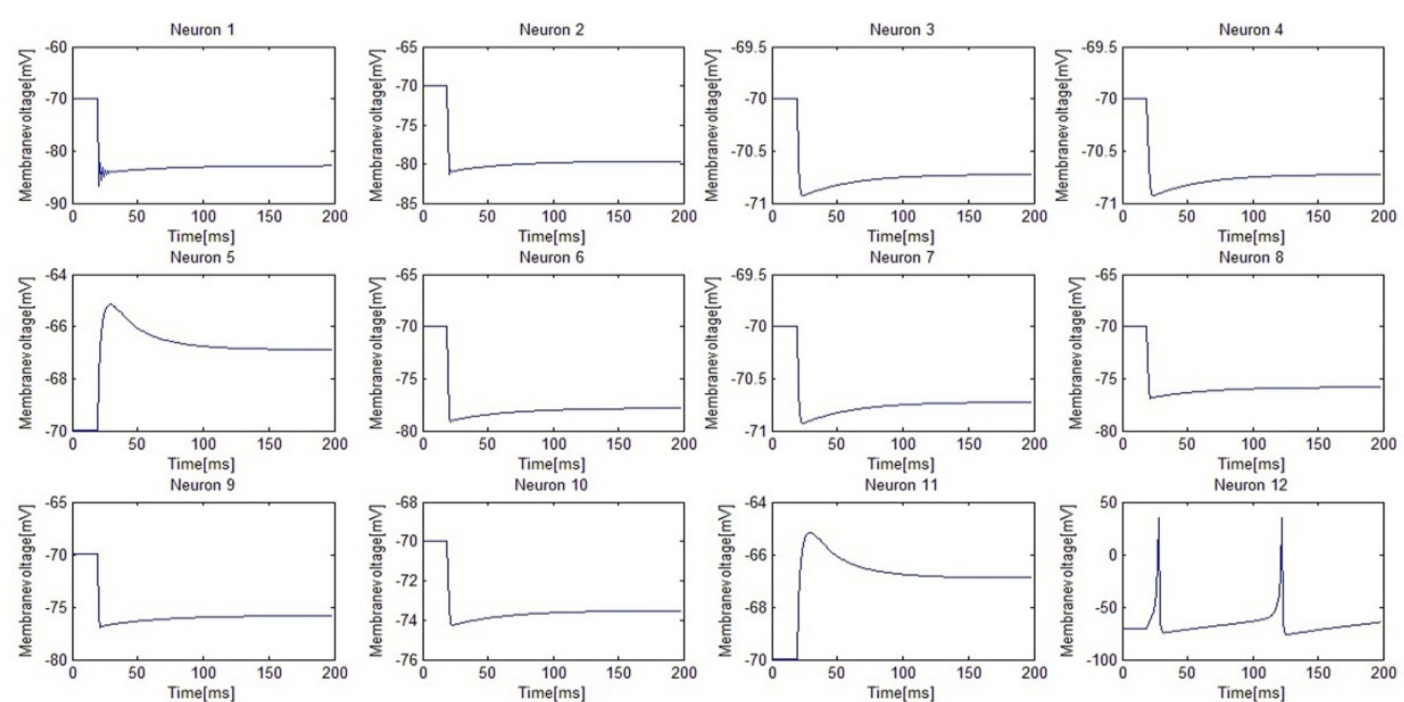

Fig.17 Behaviour of membrane voltages for character ' $x$ '

\section{REFERENCES}

[1] W. Maass, "Networks of spiking neurons: the third generation of neural network models," Neural Networks, vol. 10, no. 9, pp. 16591671, 1997.

[2] A.R. Baig , "Spatial-Temporal Artificial Neurons Applied to Online Cursive Handwritten Character Recognition," in European Symposium on Artificial Neural Networks., Bruges (Belgium)., 2004, pp. 561-566.

[3] A. Delorme and S.J. Thorpe, "SpikeNET: an event-driven simulation package for modeling large networks of spiking neurons," Network-computation in neural systems , vol. 14, 4, ) pp.613-627, 2003.

[4] A. Gupta and L. Long, "Character Recognition using Spiking Neural Networks," in International Joint Conference on Neural Networks., Orlando., Florida, USA, 2007.

[5] Y. Dan and M. Poo, "Spike time dependent plasticity of neural circuits," Neuron, vol.14, pp. 23-30, 2004.

[6] S. Thorpe, A. Delorme and R.V. Rullen, "Spike based strategies rapid processing," Neural Networks, vol. 10, pp. 715-726, 2001.

[7] D.V. Buonomano and M.M. Merzenich, "A neural network model of temporal code generation and position invariant pattern recognition," Neural Computation, vol. 11, pp.103-116, 1999.

[8] E. Izhikevich, "Which Model to Use for Cortical Spiking Neurons," IEEE Transactions on Neural Networks, vol. 15, no. 9, pp.1063$1070,2004$.

[9] E. Izhikevich, "Simple Model of Spiking Neurons," IEEE Transactions on Neural Networks, vol. 14, no. 6, pp.1569-1572, 2003.

[10] K. Rice, "Accelerating Pattern Recognition Algorithms On Parallel Computing Architectures," Ph.D. dissertation, Computer Engineering, Clemson University, 2011.

[11] A. Mohemmed, S. Schliebs, S. Matsudu and N. Kasabov, "Method for Training a Spiking Neuron to Associate Input-Output Spike Trains," in IFIP International Federation for Information Processing., Japan., 2011, pp.219-228. 
[12] F. Ponulak, "Supervised Learning in Spiking Neural Networks with ReSuMe Method Mohemmed," Ph.D. dissertation, Control and Information Engineering, Poznań University of Technology, 2006.

[13] R. Brette, "Simulation of networks of spiking neurons: A review of tools and strategies," J. Computer Neuroscience, 2006.

\section{APPENDIX}

Table below lists the important running parameters;

TABLE I Running parameters

\begin{tabular}{|c|c|}
\hline Parameter & Value \\
\hline $\mathrm{a}$ & 0.02 \\
\hline $\mathrm{b}$ & 0.2 \\
\hline $\mathrm{c}$ & -65 \\
\hline $\mathrm{b}$ & 8 \\
\hline Threshold & $30 \mathrm{mV}$ \\
\hline Step time & 1 \\
\hline$\gamma$ & 0.1 \\
\hline
\end{tabular}

